\title{
Biochemically diverse CRISPR-Cas9 orthologs
}

Giedrius Gasiunas ${ }^{1, \dagger}$, Joshua K. Young ${ }^{2, \dagger, *}$, Tautvydas Karvelis ${ }^{3}$, Darius Kazlauskas ${ }^{3}$, Tomas Urbaitis ${ }^{1,3}$, Monika Jasnauskaite ${ }^{1}$, Mantvyda Grusyte ${ }^{1}$, Sushmitha Paulraj ${ }^{2}$, Po-Hao Wang ${ }^{2,6}$, Zhenglin $\mathrm{Hou}^{2}$, Shane K. Dooley ${ }^{5}$, Mark Cigan ${ }^{2,7}$, Clara Alarcon ${ }^{2}$, N. Doane Chilcoat ${ }^{2}$, Greta Bigelyte ${ }^{3}$, Jennifer L. Curcuru ${ }^{4}$, Megumu Mabuchi ${ }^{4}$, Zhiyi Sun ${ }^{4}$, Ryan T. Fuchs ${ }^{4}$, Ezra Schildkraut ${ }^{4}$, Peter R. Weigele ${ }^{4}$, William E. Jack ${ }^{4}$, G. Brett Robb ${ }^{4, *}$, Česlovas Venclovas ${ }^{3}$, and Virginijus Siksnys ${ }^{1,3, *}$

${ }^{1}$ CasZyme, Vilnius, LT-10257, Lithuania

${ }^{2}$ Department of Molecular Engineering, Corteva Agriscience ${ }^{\mathrm{TM}}$, Johnston, IA 50131, USA

${ }^{3}$ Institute of Biotechnology, Vilnius University, Vilnius, LT-10257, Lithuania

${ }^{4}$ New England Biolabs, Ipswich, MA 01938, USA

${ }^{5}$ Department of Agricultural and Biosystems Engineering, Iowa State University, Ames, IA 50011, USA

${ }^{6}$ Present address: Inari Agriculture, West Lafayette, IN 47906, USA

${ }^{7}$ Present address: Genus plc, Deforest, WI 53532, USA

† These authors contributed equally: Giedrius Gasiunas and Joshua K. Young

* To whom correspondence should be addressed. Email: josh.young@corteva.com, robb@neb.com and siksnys@ibt.lt

\begin{abstract}
CRISPR-Cas9 nucleases are abundant in microbes. To explore this largely uncharacterized diversity, we applied cell-free biochemical screens to rapidly assess the protospacer adjacent motif (PAM) and guide RNA (gRNA) requirements of novel Cas9 proteins. This approach permitted the characterization of 79 Cas9 orthologs with at least 7 distinct classes of gRNAs and 50 different PAM sequence requirements. PAM recognition spanned the entire spectrum of T-, A-, C-, and G-rich nucleotides ranging from simple di-nucleotide recognition to complex sequence strings longer than 4. Computational analyses indicated that most of this diversity came from 4 groups of interrelated sequences providing new insight into Cas 9 evolution and efforts to engineer PAM recognition. A subset of Cas9 orthologs were purified and their activities examined further exposing additional biochemical diversity. This constituted both narrow and broad ranges of temperature dependence, staggered-end DNA target cleavage, and a requirement for longer stretches of homology between gRNA and DNA target to function robustly. In all, the diverse collection of Cas9 orthologs presented here sheds light on Cas9 evolution and provides a rich source of PAM recognition and other potentially desirable properties that may be mined to expand the genome editing toolbox with new RNA-programmable nucleases.
\end{abstract}

\section{INTRODUCTION}


The Cas9 protein from type II CRISPR (clustered regularly interspaced short palindromic repeats)-Cas (CRISPR-associated) antiviral defence systems have been repurposed as a robust genome editing tool (reviewed in $^{1-5}$ ). Target DNA specificity is accomplished with small non-coding RNAs that through direct base pairing guides Cas9 to its DNA target ${ }^{6,7}$. In addition to guide RNA (gRNA) recognition, a sequence motif, termed the PAM (protospacer adjacent motif), is required for the initiation of Cas9-guide RNA target binding and cleavage ${ }^{6,7}$. Easily reprogrammed to recognize new DNA sequences, it has been widely adopted for use in a multitude of applications to edit genomic DNA, modulate gene expression, visualize genetic loci, or detect targets in vitro ${ }^{8-15}$. To date, just a handful of variants are used for these applications ${ }^{16-18}$ with the Streptococcus pyogenes (Spy) Cas9 being used most widely ${ }^{2}$.

Since Cas 9 can be programmed to target DNA sites by altering the spacer sequence of the gRNA, recognition of the PAM becomes a constraint which restricts the sequence space targetable by Cas9. This is further limited by the requirement for careful site selection to minimize off-target binding and cleavage based on the tolerance for mismatches in the gRNA-PAM-target complex ${ }^{19-23}$. This constraint becomes particularly evident in therapeutic applications where even rare genome alterations resulting from offtargets are undesirable or when targeting more structurally complex plant genomes ${ }^{24,25}$. Moreover, these restraints impact the use of Cas9 for homology-directed repair (HDR), template-free editing, base editing, or prime editing applications, where the outcome is reliant on the proximity of the desired change to the target sequence ${ }^{26-32}$. Furthermore, the biochemical and physical characteristics of Cas9, producing predominantly blunt-end DNA target cleavage ${ }^{6,7}$, slow substrate release ${ }^{33}$, low frequency of recurrent target site cleavage ${ }^{34}$, gRNA exchangeability ${ }^{35}$, temperature dependence ${ }^{36}$, and size $^{37}$ may also be unfavourable for its varied applications.

While Spy Cas 9 targeting constraints are beginning to be addressed through structure guided rational $\operatorname{design}^{38-40}$ and directed evolution approaches ${ }^{41-44}$, we show that the diversity provided by naturally occurring orthologs may offer unique insight and opportunities for improvement of this powerful tool. First, we determined the gRNA and PAM requirements for 79 phylogenetically distinct Cas9s of various sizes without the need for protein purification or extensive computational analyses ${ }^{45}$. In doing so, we identified extraordinary diversity in Cas9 PAM and gRNA requirements. This extended the number of unique classes of gRNAs from four to seven and revealed T-, A-, C-, and G-rich PAM recognition that varied in length from one to more than four nucleotides. Interestingly, analysis of the PAM interacting (PI) domain indicated that much of this variation was derived from just 4 related groups. Finally, additional biochemical studies revealed new diversity that may further extend application. This included differences in temperature and spacer length requirements as well as variation in the pattern of doublestranded DNA target cleavage.

\section{RESULTS}




\section{Cas9 ortholog selection}

To systematically sample diversity, 47 orthologs were chosen from most of the 10 major clades of a Cas 9 evolutionary tree (Figure 1A and Supplementary Table S1). Clades giving rise to previously characterized proteins that were active in eukaryotic cells were mined at a rate of approximately $20 \%$ while all others were surveyed at a rate of approximately $10 \%$. To enrich for proteins with robust biochemical activity and thermostability, an additional 32 orthologs were selected based on their physiochemical properties (e.g. predicted secondary structure and isoelectric point), classification as a type II-A subtype ${ }^{35,46}$, and affiliation with a thermophilic host organism (Supplementary Table S2). Sequence length variation of our collection matched that found in naturally occurring orthologs and ranged from $\sim 1,000$ to $\sim 1,600$ residues with a bimodal distribution focused around sizes of $\sim 1100$ and $\sim 1375$ amino acids (Supplementary Figure S1). Furthermore, sequence alignments of those selected showed extraordinary variation, altogether, differing by as much as $93 \%$ (Supplementary Table S1).

\section{Guide RNA requirements}

In all instances, Cas9 gRNAs, the crRNA (CRISPR RNA) and tracrRNA (trans-activating CRISPR RNA), were identified near the $\operatorname{cas} 9$ gene, however, spatial positioning as well as the transcriptional orientation varied greatly among the systems characterized (Supplementary Figure S2). In general, these features were conserved among orthologs belonging to a particular phylogenetic clade (Supplementary Figure S2). Most CRISPR repeats were $\sim 36$ bp length, however, longer repeats ( $45-50 \mathrm{bp}$ ), associated with orthologs from clade X, were also identified (Supplementary Table S2). Computational analyses comparing co-variant models (CMs) based on sequence and secondary structure homology among the characterized tracrRNAs showed 7 distinct clusters (Figure 2). For some Cas9 orthologs, the tracrRNA self-clustered or demonstrated weak similarity to other CMs (Figure 2). In these cases, it was not assigned to a particular group. In general, clusters were tightly associated with a particular Cas9 phylogenetic clade, although, exceptions were noted (Figure 2). Examination of the sgRNA modules (repeat:anti-repeat duplex, nexus, and 3' hairpin-like folds ${ }^{47,48}$ were also typically conserved among related Cas 9 proteins (Supplementary Figure S3). For example, the sgRNA solutions for almost all members of clade IV resembled that belonging to Spy Cas9 and comprised a bulge in the repeat:anti-repeat duplex, a short nexus-like stem-loop, and two hairpins followed by a poly-U sequence at the 3 'end $^{47,48}$. Analogous structures were observed in the sgRNAs of Cas9 proteins belonging to clades VIII and X. However, in clade X sgRNAs, the repeat:anti-repeat duplexes were typically fully complementary and didn't form repeat:anti-repeat bulges. Members of clade V contained the shortest sgRNAs, and reminiscent of the sgRNA from Streptococcus aureus (Sau) Cas9, these contained only 2 hairpins (nexus-like followed by a larger fold) following the repeat:anti-repeat duplex (Supplementary Figure S3). In contrast, sgRNAs associated with clades III, VI, and IX orthologs displayed longer, more complex and diverse structures. These included a variety of differences in stem length, presence of bulges, and different spacing between 
sgRNA modules (Supplementary Figure S3). In addition, it was more difficult to reliably identify a Rhoindependent-like terminator at the end of some tracrRNA encoding regions for these clades.

\section{PAM recognition by orthologous Cas9s}

To rapidly survey the target recognition properties of new Cas9s, we employed a cell-free in vitro translation (IVT) method similar to that described previously (Figure 1B) ${ }^{45,49}$. Since PAM recognition is dependent on the concentration of Cas9-guide RNA complex ${ }^{50}$, crude IVT RNP mixtures were diluted ( $10^{1}$ to $10^{3}$ in 10 -fold increments) and tested for their ability to support cleavage when combined with a plasmid library containing a randomized PAM region adjacent to a Cas 9 target site. The greatest dilution supporting cleavage activity was then used as a baseline for PAM recognition. To confirm the accuracy of our approach, Cas9 PAM recognition was examined using purified components as described previously ${ }^{50}$. This was done for Spy, S. thermophilus CRISPR3 (Sth3), and S. thermophilus CRISPR3 (Sth1) Cas9s, whose PAM was determined previously ${ }^{50}$ and for 11 orthologs from our collection. As shown in Supplementary Figure S4A \& B, there was a nearly perfect agreement between the approaches. Additionally, to examine the propensity for PAM recognition to extend beyond position 7 (the length of randomization in our PAM library), the spacer targeting the PAM library was also shifted 5' by 1, 2 or 3 nts for Cas9 orthologs that exhibited PAM preferences at positions 6 or 7 and lacked PAM requirements in the first, second or third positions. This permitted PAM identification to be extended to 8, 9, or $10 \mathrm{bp}$, respectively. Of the 20 Cas9s that were tested (Supplementary Table S2), only 5, all belonging to phylogenetic clade VI (Figure 1A \& 2), had PAM recognition that continued beyond the $7^{\text {th }}$ position. Surprisingly, PAM preferences at the $8^{\text {th }}$ position were always an A residue similar to the previously characterized Brevibacillus laterosporus (Blat) ${ }^{50}$ and Geobacillus stearothermophilus $(\mathrm{Geo})^{51} \mathrm{Cas} 9$ proteins. Altogether, we found that long PAMs extending beyond the $7^{\text {th }}$ position were not widespread and only abundant in one family of orthologs belonging to clade VI.

The Cas9 orthologs characterized (Supplementary Table S2) with our IVT-based approach demonstrated significant divergence in PAM recognition. Indeed, we identified nucleases with novel PAM requirements that varied in composition both in sequence and length. Among these were proteins with PAM recognition that could be generally sub-divided into A-, T-, and C-rich PAM recognition in addition to the G-rich PAM typical of the Spy Cas9 protein (Figure 3). PAMs composed of multiple residues of a single base pair while present (e.g. Efa, Nme2, Rsp, Ssi, and Ssu) were rare but notably enriched in Clade IV (e.g Efa) to which Spy Cas9 belongs, and in Clade VII (Ssi, Ssu) (Figure 1A, Figure 3, and Supplementary Table S3). In general, Cas9s with composite PAM recognition containing at least two different base pairs were more abundant (Figure 3). The length of PAM recognition also varied between 1-4 base pairs or more with most orthologs exhibiting recognition at three or more positions.

Additionally, many proteins exhibited seemingly degenerate PAM recognition. Typically, this resulted in 
a strong requirement for at least one base pair in combination with positions that accepted more than one (typically two) base pairs (e.g. Lan, Mse, Nsa, and Sma).

\section{Diversity and taxonomic distribution of Cas9 PAM interacting domains}

The extreme diversity of experimentally determined PAM sequence requirements prompted us to evaluate the sequence relationship of Cas9 PAM interacting (PI) domains. To do so, we extracted the PI regions from the characterized orthologs and used them as queries for iterative searches against non-redundant collections of microbial proteins (see methods). In all, 9,161 sequences having non-identical PI domains were found (Supplementary Table S4). Sequences were next clustered based on their pairwise similarity leading to identification of ten clusters (Figure 3). Clusters 1-4 were the largest and contained $93 \%$ of all sequences recovered while clusters 7-10 were considerably smaller and were comprised of 4 to 37 sequences (Figure 3, Supplementary Table S4). Sequence searches with HHpred ${ }^{52}$ showed that most clusters were distantly related to each other (Figure 3 and Supplementary Figure S5, A-C) with an exception being cluster 10 that did not reveal significant similarity to any other group (Supplementary Figure S5, D). In general, PI domain similarity could be correlated with the major phylogenetic branches of the Cas 9 tree (Figure 1A and Figure 3). For example, Cas9s belonging to clades II, III, and IV grouped into cluster 1 (Figure 3). Additionally, phylogenetic analysis of clusters 1-6 also suggested that similar PI domains usually resulted in similar PAM recognition (Supplementary Figure S6 A, B, D), however, sequence diversity and length varied greatly even among members of the same group (Supplementary Figure S6C). Closer examination of the Cas9s belonging to cluster 1 further highlighted that even within similar PI architectures sequence composition varied considerably with conservation being the lowest in the PI domain relative to the rest of the Cas9 protein (Supplementary Figure S7).

Although clusters shared amino acid sequence similarity (Figure 3), their taxonomic distribution differed. While PI domains from cluster 2 were mainly found in Bacteroidetes and Alphaproteobacteria (Supplementary Figure S6B), cluster 3 was more likely to come from Betaproteobacteria, Epsilonproteobacteria, and Firmicutes (Bacilli and Clostridia) (Supplementary Figure S6C). Sequences from clusters 1 and 4 were usually found in Firmicutes (Supplementary Figure S6 A \& D) while clusters 5 and 6 were specific to Actinobacteria and Proteobacteria, respectively (Supplementary Figure S5 E).

\section{Evaluation of Cas9 ortholog biochemical activity}

Fifty-two Cas 9 orthologs from our collection were selected for additional characterization using purified components. Primary selection criteria included simple PAM recognition $(\leq 3 \mathrm{bp})$ (where possible) while maintaining diversity in phylogenetic distribution and protein size. It was previously reported that Sau and Geo Cas9 proteins require a longer spacer to function robustly ${ }^{16,51}$. Therefore, we designed sgRNAs with two different spacer lengths, 20 and $24 \mathrm{nt}$, for each ortholog and examined their influence on Cas9 
cleavage activity in vitro. Exceptions to this included Efa, Lpn and Cme4 where a single spacer length of either 20 or $22 \mathrm{nt}$ was tested. As shown in Supplementary Figure S8, most orthologs worked best with a $20 \mathrm{nt}$ spacer similar to Spy Cas9 when evaluated across a panel of 5 different buffers, however, six orthologs, Cga, Cca1, Orh, Tmo, Nsa, and Ghh1 Cas9, required a spacer length of greater than $20 \mathrm{nt}$ to effectively cut their DNA target. In all, 46 out of 52 produced double-stranded DNA target cleavage activity greater than $25 \%$ under the conditions examined.

The thermal stability of thirty-eight orthologs showing robust target cleavage were next predicted using nano differential scanning fluorimetry (nanoDSF). 36 of 38 proteins showed a melting temperature of $>37^{\circ} \mathrm{C}$ confirming stability under standard in vitro enzymatic reaction conditions. Interestingly, five orthologs had melting temperatures $>50^{\circ} \mathrm{C}$ suggesting thermostability (Supplementary Figure S9). These included Cme2, Cme4, Ghy1, Esp1 and Nsa Cas9.

To corroborate nanoDSF predictions, DNA target cleavage was next measured in reactions at temperatures ranging from $10^{\circ} \mathrm{C}$ to $70^{\circ} \mathrm{C}$. In all, Cas 9 orthologs displayed a wide spectrum of temperature dependencies including both narrow and broad ranges of activity (Figure 4A). Consistent with thermal unfolding analysis, Cme2, Esp1, Nsa, Ain, Cme3, and Sth1A, were active at temperatures greater than $50^{\circ} \mathrm{C}$ with $\mathrm{Nsa}$, isolated from the deep-sea hydrothermal vent chimney bacterium, Nitratifractor salsuginis ${ }^{53}$, remaining active at temperatures greater than $60^{\circ} \mathrm{C}$ (Figure 4B). Additionally, one ortholog, Ssa, retained $95 \%$ of its cleavage activity at $10^{\circ} \mathrm{C}$ (Figure $4 \mathrm{~A}$ ). We also observed that 5 Cas 9 orthologs (Cme2, Cme4, Nsp, Khu, and Fma) retained less than $25 \%$ activity at reaction temperatures of $25^{\circ} \mathrm{C}$ or below.

\section{Target DNA cleavage by Cas9 orthologs}

To characterize the termini resulting from Cas9 DNA cleavage, we developed a method which allows both termini resulting from target cleavage to be captured simultaneously in a deep sequencing read (Supplementary Figure S10). To validate the approach, we examined the cleavage positions for restriction endonucleases HhaI, FspI, and HinP1I, and for Spy Cas9. As shown in Figure 5A, these enzymes generated defined cut-sites, either blunt-ended or staggered as expected. This can be contrasted with Spy Cas 9 target cleavage that, depending on target site, generated either blunt-end cuts, $1 \mathrm{nt} 5$ ' staggered termini due to RuvC post-cleavage trimming as observed previously ${ }^{54}$, or a mixture of both (Supplementary Figure S11). In all, as averaged across 5 targets, Spy Cas9 generated predominantly blunt-ended DNA target cleavage as reported previously ${ }^{7}$ (Figure 5B). Analysis of Sau Cas 9 target cleavage produced almost entirely blunt-ended products as shown earlier ${ }^{54}$ (Figure 5B and S11 and Supplementary Table S5). 
We next evaluated the target cleavage pattern for 19 orthologs from our collection. Some Cas9s produced blunt-ended termini like Sau Cas9 (e.g. Cpe and Tsp (Fig 4C)) while others, depending on the target site, produced either blunt-ended or $1 \mathrm{nt} 5$ '-overhang termini similarly to Spy Cas9 (e.g. Sag1 and Seq1 (Supplementary Table S5)). Some orthologs, as averaged across 5 different target sites, consistently generated 5'-overhang termini varying between 1 or more nts (e.g. Khu, Lpn, Nsa, and Esp1) (Figure 5C and Supplementary Table S5). In these cases, the non-target strand tended to terminate at multiple positions suggesting variation in the positioning of or post-cleavage trimming by the RuvC domain while the target strand was cleaved predominantly between the $3^{\text {rd }}$ and $4^{\text {th }}$ positions of the protospacer (Figure 5C and Supplementary Table S5).

\section{DISCUSSION}

We identified Cas9s with novel G-rich, C-rich, A-rich, and T-rich PAM recognition of varying compositions, altogether, greatly expanding the sequence space targetable by Cas 9 . The observed diversity in PAM length was also striking with the majority of orthologs recognizing PAMs greater than 2 bp. This difference may be important for genome editing applications as orthologs with longer PAM recognition ( $\geq 3 \mathrm{bp}$ ) may afford higher specificity ${ }^{16,17,55}$. Additionally, phylogenetic and clustering analyses revealed that the PI domain was not always congruent with the rest of the protein. For example, conservation of the PI domain among related Spy Cas9 proteins was 1.4 times lower relative to the Nterminal portion (Supplementary Figure S7). In some cases, these differences were even greater as was noted for orthologs from Neisseria meningitidis (Nme) where PI domains only shared 52\% identical residues while the rest of the protein was nearly the same $(98 \% \text { identity })^{56}$ or in clade $\mathrm{X}$ where sequences belonged to three different homology clusters (Figure 3; clusters 7, 5 and 10). Moreover, sequence variation from clusters 1, 3 and 4 when compared with the structures of Spy (4zto), Nme (6kc8), Sau (5axw) and Sth (6rjd) could be modelled into just a single PI domain architecture (Supplementary Figure S12). Altogether, these observations could in part be explained by the uncoupling of PI domain evolution from the rest of protein indicating that it is under selective pressure to diversify perhaps in response to PAM-based phage escape strategies as described previously ${ }^{57-59}$. Additionally, they suggest that the Cas9 PI domain is extraordinarily flexible and can be engineered to recognize a wide variety of sequence motifs encompassing the full spectrum of DNA nucleotides.

The gRNAs from our collection were in general conserved between related Cas9 proteins, although, new and diverse gRNA structures were observed. In general, they could be classified into 7 groups based on tracrRNA sequence and structural homology and visual inspection of sgRNA modules as exemplified by Seq1 (Spy-like), Cca1, Tde, Sth1A, Lsp1, Nme2, and Gsp (Figure 2 and Supplementary Figure S3). Altogether, this may warrant the expansion of the number of discrete non-cross reactive Cas 9 and sgRNA 
combinations from 4 to 7 or more pending future studies ${ }^{35,60}$. This finding is important for orthogonal genome editing approaches where simultaneous, yet disparate activities are required at different sites ${ }^{60,61}$.

Finally, an in-depth evaluation of DNA cleavage activity of the Cas9 nucleases described here exposed additional differences among orthologs. These included a wide range of temperature dependencies. Of particular interest was $\mathrm{Cme} 2 \mathrm{Cas} 9$, which was only robustly active from $\sim 30^{\circ} \mathrm{C}$ to $55^{\circ} \mathrm{C}$ suggesting the possibility of temperature-controlled DNA search and modification. Additionally, the DNA cleavage activity at different temperatures for Nsa and Ssa Cas9s suggested they could be harnessed for use in thermo- or psychrophiles, respectively. Furthermore, we characterized orthologous Cas9 nucleases with different, and potentially advantageous properties compared to those generally prescribed to Spy Cas9. These included variation in the termini resulting from target cleavage as well as a preference for a longer tract of gRNA and DNA target site homology.

\section{METHODS}

\section{Identification and phylogeny of Cas9 orthologs}

Type II Cas9 endonucleases were identified by searching for the presence of an array of CRISPRs using PILER-CR ${ }^{62}$. Following identification, the DNA sequences surrounding the CRISPR array (about $15 \mathrm{~kb}$ 5' and 3' of the CRISPR array) were examined for the presence of open-reading frames (ORFs) encoding proteins greater than 750 amino acids. Next, to identify cas genes encoding Cas 9 orthologs, multiple sequence alignment of sequences from a diverse collection of Cas9 proteins was performed using MUSCLE ${ }^{63}$ and then used to build profile hidden Markov models (HMMs) for Cas9 sub-families as described previously ${ }^{35}$ using HMMER $^{64,65}$. The resulting HMMs were then utilized to search protein sequences translated from the cas ORFs for the presence of genes with homology to Cas9. Alternatively, Cas9 orthologs and the metagenomic sequence encoding them were obtained from publicly available datasets through the Joint Genome Institute's Integrated Microbial Genomes \& Metagenomes resource (IMG/M): https://img.jgi.doe.gov/cgi-bin/m/main.cgi ${ }^{66}$. Only proteins containing the key HNH and RuvC nucleolytic domains and catalytic residues defining a type II Cas 9 protein ${ }^{67}$ were selected (Supplementary Table S6). Through phylogenetic analyses $\left(M E G A 7^{68}\right.$ ) Cas9 proteins were then parsed into distinct families and representative members of each group used to select orthologs for characterization. To place our collection in context with previously described Cas9 orthologs, a phylogenetic tree was built using type II-A, $-\mathrm{B}$, and $-\mathrm{C}$ representatives ${ }^{69}$ and those we selected for characterization using MEGA $7{ }^{68}$ employing Neighbor-Joining ${ }^{70}$ and Poisson correction ${ }^{71}$ methods.

\section{Engineering single guide RNA solutions}

The trans-activating CRISPR RNA (tracrRNA) essential for CRISPR RNA (crRNA) maturation ${ }^{72}$ and Cas9 directed target site cleavage in type II systems ${ }^{7,73}$ was identified by searching for a region in the 
vicinity of the $\operatorname{cas} 9$ gene, the anti-repeat, which may base-pair with the CRISPR repeat and was distinct from the CRISPR array(s). Once identified, the possible transcriptional directions of the putative tracrRNAs for each new system were established by examining the secondary structures using UNAfold ${ }^{74}$ and VARNA ${ }^{75}$ and possible termination signals present in RNA versions corresponding to the sense and anti-sense transcription scenarios surrounding the anti-repeat. Based on the likely transcriptional direction of the tracrRNA and CRISPR array, single guide RNAs (sgRNAs), representing a fusion of the CRISPR RNA (crRNA) and tracrRNA ${ }^{7}$, were designed. For each ortholog, this was accomplished by linking $16 \mathrm{nt}$ of the crRNA repeat to the complementary sequence of the tracrRNA anti-repeat by a 4 nt GAAA loop similar to that described previously for Spy Cas $9^{7}$. All repeat, tracrRNA sequences and sgRNA solutions are listed in Supplementary Table S2.

\section{Computational analysis of Cas9 tracrRNAs}

BLAST (with parameters to optimize finding short sequences in highly-repetitive regions (-task blastn_short -dust no) $)^{76}$ was used to identify sequences homologous to the 79 identified tracrRNAs. The resulting collection of identified sequences were grouped using $\mathrm{CD}-\mathrm{HIT}^{77}$ at a $90 \%$ sequence similarity threshold. The resulting clusters were filtered to remove groups that did not contain at least one of the 79 reference tracrRNA sequences. Next, sequence homology and secondary structure models were constructed for each group using $\mathrm{MAFFT}^{78}$ and RNAalifold ${ }^{79}$, respectively. Both models were then used to search for sequence/structural homology in the full set of reference and BLAST-identified sequences using the RNA structure search tools in the Infernal v1.1 software suite ${ }^{80}$. Structural overlap between clusters was then generated by comparing the results of each covariance model (CM). To graph the relationship among tracrRNAs, vertices were first added for each representative CM (sequences with both shared secondary structure predictions and at least $90 \%$ sequence similarity). If two vertices shared a CM, they were connected with a line weighted by the percent similarity between shared vertices (percent similarity $=(\#$ of shared sequences $) /(\min$ (\# found by model 1 , \# found by model 2$))$ ).

\section{Production of single guide RNAs}

All single guide RNA (sgRNA) molecules used in this study were synthesized by in vitro transcription using HiScribe ${ }^{\text {TM }}$ T7 Quick High Yield RNA Synthesis Kits (New England Biolabs), or transcribed directly in the in vitro translation (IVT) reaction. Templates for sgRNA transcription were generated by PCR amplifying synthesized fragments (IDT and Genscript) or by annealing a T7 primer oligo to a single stranded template oligonucleotide. Transcribed RNA products were treated with DNaseI (New England Biolabs) to remove DNA templates and cleaned up with Monarch RNA Cleanup Kit (50 $\mu$ g) (New England Biolabs) and eluted in nuclease-free water. RNA concentration and purity were measured by NanoDrop spectrophotometry and RNA integrity was visualized by SYBR ${ }^{\mathrm{TM}}$ Gold staining of reaction 
products separated on Novex TBE-Urea $15 \%$ denaturing polyacrylamide gels with $0.5 \mathrm{x}$ TBE (Tris borate EDTA) buffer.

\section{PAM library cleavage using in vitro translation}

Cas9 was produced by IVT using either a continuous exchange 1-Step Human Coupled IVT Kit (Thermo Fisher Scientific) or a PURExpress bacterial IVT kit (New England Biolabs), following the manufacturer's recommended protocol similar to that described previously ${ }^{45}$. Plasmid DNA encoding human or E. coli codon optimized Cas9s were generated for use as templates for IVT reactions. Synthetic DNA fragments were synthesized by Genscript, Inc. and Twist Bioscience and assembled by NEBuilder HiFi DNA Assembly kit (New England Biolabs) into pT7-N-His-GST (Thermo Fisher Scientific) or pET28a (EMD Millipore). Following IVT, $20 \mu$ of supernatant containing soluble Cas9 protein was mixed with RiboLock RNase Inhibitor (40 U; Thermo Fisher Scientific) and $2 \mu \mathrm{g}$ of T7 in vitro transcribed sgRNA and incubated for $15 \mathrm{~min}$. at room temperature. Alternatively, the sgRNA was transcribed directly in the IVT kit by supplying a DNA template containing a T7 promoter and sequence encoding the respective sgRNA. In this situation, $0.5 \mu \mathrm{g}$ of plasmid encoding the cas 9 gene and a 100fold molar excess of sgRNA template was added to the IVT reaction mix. $10 \mu \mathrm{l}$ (or series of ten-fold dilutions) of the resulting Cas9-sgRNA ribonucleoprotein (RNP) complex were then combined with $1 \mu \mathrm{g}$ of the $7 \mathrm{bp}$ randomized PAM library described previously ${ }^{50}$ in a $100 \mu \mathrm{l}$ reaction buffer $(10 \mathrm{mM}$ Tris- $\mathrm{HCl}$ pH 7.5 at $37^{\circ} \mathrm{C}, 100 \mathrm{mM} \mathrm{NaCl}, 10 \mathrm{mM} \mathrm{MgCl} 2,1 \mathrm{mM} \mathrm{DTT}$ ) and incubated for $60 \mathrm{~min}$. at $37^{\circ} \mathrm{C}$. Finally, cleaved library fragments were captured by adapter ligation, enriched for by PCR amplification, and deep sequenced as described previously ${ }^{50}$.

\section{Identification of PAM preferences}

PAM sequences that supported double-stranded DNA target cleavage were captured by adapter ligation, enriched for by PCR amplification, and deep sequenced as described earlier ${ }^{45,50,81}$. Bias in the bp composition at each position within the randomized PAM library was first adjusted to that in the starting library by normalization ((Treatment Frequency)/((Control Frequency)/(Average Control Frequency))). Then, PAM preferences were quantified using position frequency matrices (PFMs) ${ }^{82}$ and displayed as a WebLogo ${ }^{83}$. Analyses were limited to the top $10 \%$ most frequent PAMs to reduce the impact of background noise resulting from non-specific cleavage coming from other components in the IVT mixtures.

\section{Computational analysis of Cas9 PAM interacting domains}

The Cas9 orthologs characterized here were aligned using MAFFT $^{78}$. Their PAM interacting (PI) regions corresponding to the C-terminal domain of Streptococcus pyogenes Cas9 (4ZT0_A:1090-1365) were extracted and used as queries for two iterations of PSI-BLAST ${ }^{84}$ search against the NCBI NR protein 
collection, UniRef100 and MGnify ${ }^{85}$ databases. Hits were extracted, filtered to $80 \%$ identity using CDHIT $^{77}$ and clustered with CLANS ${ }^{86}$. Resulting networks were visually inspected and clusters identified. For groups larger than 150 sequences (Supplementary Table S4), a phylogenetic analysis was performed recovering sequences which were filtered out during the previous step and removing identical ones. Next, multiple sequence alignments were performed for clusters 1 to 6 using MAFFT (options: "--ep 0.123 -maxiterate 20 --localpair") and regions with gaps removed with trim $\mathrm{AL}^{87}$ (option: "-gt 0.01 "). Lengths of the resulting alignments varied from 359 to 652 residues in clusters 2 and 3, respectively. Phylogenetic trees were generated using IQtree ${ }^{88}$ with auto model selection and 1000 fast bootstrap (options: "-alrt 1000 -bb 1000").

\section{Cas9 expression and purification}

Spy, S. thermophilus CRISPR3 (Sth3), and S. thermophilus CRISPR3 (Sth1) Cas9 proteins were expressed and purified as described previously ${ }^{50}$. Other orthologs were first $E$. coli codon optimized and cloned into the pET28 vector, to yield constructs encoding fusion proteins comprising a C-terminal 6-Histag. In some instances, sequences encoding nuclear localization sequences (SV40 origin) were incorporated onto the 5' and 3' ends of the $\operatorname{cas} 9$ gene. The expression analysis was then performed in different E. coli strains (NiCo21(DE3), T7 Express lysY/Iq, NEB® Express Iq) under various growth conditions (media, temperature, induction) and detected by SDS-PAGE analysis. Optimised conditions then were chosen for flask scale purification. Cells were disrupted by sonication. The supernatant was loaded onto HiTrap DEAE Sepharose (GE Healthcare), followed by subsequent purification on $\mathrm{Ni}^{+}{ }^{+}$ charged HiTrap chelating HP column (GE Healthcare) and HiTrap Heparin HP (GE Healthcare) columns. Purified Cas9 proteins were stored at $-20^{\circ} \mathrm{C}$ in $20 \mathrm{mM}$ Tris- $\mathrm{HCl}$, $\mathrm{pH}$ 7.5, $500 \mathrm{mM} \mathrm{KCl,} 1 \mathrm{mM}$ EDTA, 1 $\mathrm{mM}$ DTT, and 50\% (v/v) glycerol.

\section{Evaluation of protospacer cleavage patterns}

To capture protospacer cleavage patterns with single molecule resolution, we developed a minicircle double stranded (ds) DNA substrate that allows both ends of target cleavage to be captured in a single Illumina sequence read. First, 124 nt oligonucleotides (IDT) (see Supplementary Table S7) were circularized using with CircLigase ${ }^{\mathrm{TM}}$ single stranded (ss) DNA Ligase (Lucigen) according the manufacturers suggestion. Circularized ssDNA was next purified and concentrated using a Monarch ${ }^{\circledR}$ PCR \& DNA Cleanup Kit (NEB). 20 pmol of the purified product was then incubated with 25 pmol of a complementary primer in $1 \mathrm{X}$ T4 DNA ligase buffer (NEB) supplemented with $40 \mu \mathrm{M}$ dNTPs. To allow the primer to anneal, the reaction was then heated to $65^{\circ} \mathrm{C}$ for 30 seconds followed by a decrease in temperature to $25^{\circ} \mathrm{C}$ at a rate of $0.2^{\circ} \mathrm{C} /$ second. 6 units of T4 DNA polymerase and 400 units of T4 DNA ligase (NEB) were then added and the reaction was incubated at $12^{\circ} \mathrm{C}$ for 1 hour to allow second strand synthesis. Following purification with a Monarch ${ }^{\circledR}$ PCR \& DNA Cleanup Kit and elution into $1 \mathrm{X}$ 
CutSmart ${ }^{\circledR}$ buffer (NEB) containing 1 mM ATP, 15 units of Exonuclease V (RecBCD; NEB) and T5 exonuclease (NEB) were added to the sample and incubated at $37^{\circ} \mathrm{C}$ for $45 \mathrm{~min} .0 .04$ units of Proteinase $\mathrm{K}$ (NEB) was then added and the sample was incubated at $25^{\circ} \mathrm{C}$ for $15 \mathrm{~min}$. prior to purification with a Monarch ${ }^{\circledR}$ PCR \& DNA Cleanup Kit. After elution, the yield of circular double-stranded DNA was assessed using an Agilent 2100 Bioanalyzer.

For minicircle digestion, Cas9 RNPs were formed by incubating 1 pmol of sgRNA with 0.5 pmol of Cas9 protein in $1 \mathrm{X}$ NEBuffer ${ }^{\mathrm{TM}} 3.1$ or 2.1 (NEB) at room temperature for $10 \mathrm{~min}$. $0.1 \mathrm{pmol}$ of circular dsDNA substrate was added, samples were incubated for $15 \mathrm{~min}$ at $37^{\circ} \mathrm{C}$ and then each $20 \mu 1$ reaction was quenched by the addition of $5 \mu 1$ of 0.16 M EDTA. Reactions were concentrated and purified with a Monarch ${ }^{\circledR}$ PCR \& DNA Cleanup Kit and the entire $8 \mu$ l of eluted product was used as a substrate for Illumina sequencing library construction using a NEBNext ${ }^{\circledR}$ Ultra $^{\mathrm{TM}}$ II DNA Library Prep Kit for Illumina ${ }^{\circledR}(\mathrm{NEB})$ and the protocol provided with the kit. 15 cycles of PCR were used to add the Illumina priming sequences and index barcodes and then the concentration of each reaction was assessed on an Agilent 2100 Bioanalyzer. Libraries were pooled and sequenced on either an Illumina NovaSeq or NextSeq instrument with 2 X 150 paired-end sequencing runs. Cleavage sites were then mapped using custom scripts and visualized as heatmaps (representing proportion) cleaved using GraphPad Prism 8.

\section{In vitro cleavage assays for determining optimal buffer, temperature, and spacer length}

First, DNA substrates containing a canonical PAM for each ortholog were amplified from HEK293T genomic DNA by PCR using primers corresponding to WTAP and RUNX1. Forward primers were labelled with 5'-FAM and 5'-ROX for WTAP and RUNX1, respectively. Reverse primers were unlabelled. 515 and 605 bp PCR products for WTAP and RUNX1, respectively, were then purified with a Monarch ${ }^{\circledR}$ PCR \& DNA Cleanup Kit ( $\left.5 \mu \mathrm{g}\right)$ (NEB T1030S) and DNA concentration and purity measured by NanoDrop ${ }^{\mathrm{TM}}$ spectrophotometry (ThermoFisher). Purified Cas9 protein was then diluted to $1 \mu \mathrm{M}$ in dilution buffer (300mM NaCl, 20mM Tris, pH7.5) and stored on ice. Next, sgRNAs (Supplementary Table S2 and S7) were diluted to $2 \mu \mathrm{M}$ in nuclease-free water. Cas 9 and $\operatorname{sgRNA}$ were then combined in a 2:1 sgRNA:Cas9 molar ratio in reaction buffer at room temperature for $10 \mathrm{~min}$. Substrate was added next at a Cas9:sgRNA:DNA ratio of 10:20:1 and incubated for $30 \mathrm{~min}$. For buffer optimization and spacer length preference experiments, 1X NEBuffers 1.1, 2.1, 3.1, or CutSmart (NEB B7200S) were used as reaction buffers and incubations took place at $37^{\circ} \mathrm{C}$. For thermoactivity experiments, reactions were performed in NEBuffer 3.1. Here, RNPs were initially formed at room temperature and then transferred to a thermal cycler pre-heated or cooled to the various assay temperatures prior to DNA substrate addition. 10x DNA substrate $(100 \mathrm{nM})$ was separately equilibrated at the designated temperature prior to being added to the RNP containing reaction tubes. Reactions were quenched by adding SDS to $0.8 \%(\mathrm{v} / \mathrm{v})$ and $80 \mathrm{mU}$ Proteinase K (NEB P8107S). Cleavage products were diluted 4X in nuclease-free water and 
subjected to capillary electrophoresis (CE) to quantify the extent of cleavage ${ }^{89}$. The fraction of substrate cleaved at each temperature was then visualized as heatmaps, using GraphPad Prism 8.

\section{Cas9 protein thermal stability}

Purified Cas9 proteins were diluted in $300 \mathrm{mM} \mathrm{NaCl}, 20 \mathrm{mM}$ Tris, $\mathrm{pH} 7.5$ to $5-10 \mu \mathrm{M}$ at room temperature. $10 \mu \mathrm{L}$ of the diluted protein was loaded into NanoDSF Grade Standard Capillaries (NanoTemper) and melting temperatures were determined using a Prometheus NT4.8 NanoDSF instrument according to the manufacturer's instruction. Temperature was increased from $20^{\circ} \mathrm{C}$ to $80^{\circ} \mathrm{C}$ at the rate of $1^{\circ} \mathrm{C} / \mathrm{min}$. Inflection points of melting curves are reported as the $\mathrm{Tm}^{90}$.

\section{DATA AVAILABILITY}

Raw deep sequencing data that support PAM and cleavage pattern determination for Cas9 orthologs were deposited at XXXX under Accession numbers XXXX. All other relevant data are available from the corresponding authors on reasonable request.

\section{ACCESSION NUMBERS}

\section{ACKNOWLEDGEMENT}

We thank Migle Stitilyte from CasZyme for preparation of the sgRNA templates.

\section{CONRIBUTIONS}

G.G., J.K.Y., M.C., C.A., N.D.C., W.E.J., E.S., G.B.R. and V.S. designed research; G.G., J.K.Y., T.K., D.K., T.U., M.J., M.G., S.P., P.W., Z.H., S.K.D., G.B., J.L.C., M.M., Z.S., R.F.T., and P.R.W. performed research, and G.G., J.K.Y., C.A., N.D.C., W.E.J., E.S., G.B.R., C.V. and V.S. analyzed data. G.G., J.K.Y., G.B.R. and V.S. wrote the paper. All authors read and approved the final manuscript.

\section{CONFLICT OF INTEREST}

Z.H., J.K.Y., G.G and V.S. have filed patent applications related to the manuscript. G.G, T.U, M.J. and M.G. are employees of CasZyme. J.K.Y., S.P., Z.H., C.A., and N.D.C. are employees of Corteva Agriscience. J.L.C., M.M., R.T.F, E.S., P.R.W., Z.S., W.E.J. and G.B.R. are employees of NEB. V.S. is a Chairman of CasZyme. V.S. and G.G. have financial interest in CasZyme.

\section{SUPPLEMENTARY INFORMATION}

Supplementary Figure S1-S12. 


\section{Supplementary Table S1}

Cas9 protein identity matrix

Supplementary Table S2

List of analysed Cas9 protein, gene, protein, and sgRNA sequences.

\section{Supplementary Table S3}

Position frequency matrices (PFM) of Cas9 ortholog PAM recognition

Supplementary Table S4

List of PAM interaction domain sequences and cluster information.

\section{Supplementary Table S5}

Target DNA cleavage patterns produced by Cas 9 orthologs

\section{Supplementary Table S6}

List of Cas9 protein sequences used to construct the phylogenetic tree.

\section{Supplementary Table S7}

Sequences of oligonucleotides and substrates used for this study. 


\section{REFERENCES}

1. Adli, M. The CRISPR tool kit for genome editing and beyond. Nat. Commun. 9, 1911 (2018).

2. Barrangou, R. \& Doudna, J. A. Applications of CRISPR technologies in research and beyond. Nat. Biotechnol. 34, 933-941 (2016).

3. Hsu, P. D., Lander, E. S. \& Zhang, F. Development and Applications of CRISPR-Cas9 for Genome Engineering. Cell 157, 1262-1278 (2014).

4. Knott, G. J. \& Doudna, J. A. CRISPR-Cas guides the future of genetic engineering. Science 361, 866-869 (2018).

5. Wang, H., La Russa, M. \& Qi, L. S. CRISPR/Cas9 in Genome Editing and Beyond. Annu. Rev. Biochem. 85, 227-264 (2016).

6. Gasiunas, G., Barrangou, R., Horvath, P. \& Siksnys, V. Cas9-crRNA ribonucleoprotein complex mediates specific DNA cleavage for adaptive immunity in bacteria. Proc. Natl. Acad. Sci. U. S. A. 109, E2579-2586 (2012).

7. Jinek, M. et al. A programmable dual-RNA-guided DNA endonuclease in adaptive bacterial immunity. Science 337, 816-21 (2012).

8. Chen, B. et al. Dynamic Imaging of Genomic Loci in Living Human Cells by an Optimized CRISPR/Cas System. Cell 155, 1479-1491 (2013).

9. Cong, L. et al. Multiplex Genome Engineering Using CRISPR/Cas Systems. Science 339, 819823 (2013).

10. Gilbert, L. A. et al. CRISPR-Mediated Modular RNA-Guided Regulation of Transcription in Eukaryotes. Cell 154, 442-451 (2013).

11. Gilbert, L. A. A. et al. Genome-Scale CRISPR-Mediated Control of Gene Repression and Activation. Cell 159, 1-15 (2014).

12. Jinek, M. et al. RNA-programmed genome editing in human cells. Elife 2, e00471 (2013).

13. Mali, P. et al. RNA-Guided Human Genome Engineering via Cas9. Science 339, 823-826 (2013).

14. Ran, F. A. et al. Double Nicking by RNA-Guided CRISPR Cas9 for Enhanced Genome Editing Specificity. Cell 1-10 (2013). doi:10.1016/j.cell.2013.08.021

15. Hajian, R. et al. Detection of unamplified target genes via CRISPR-Cas9 immobilized on a graphene field-effect transistor. Nat. Biomed. Eng. 3, 427-437 (2019).

16. Ran, F. A. et al. In vivo genome editing using Staphylococcus aureus Cas9. Nature 520, 186-191 (2015).

17. Müller, M. et al. Streptococcus thermophilus CRISPR-Cas9 Systems Enable Specific Editing of the Human Genome. Mol. Ther. 24, 636-644 (2016).

18. Kim, E. et al. In vivo genome editing with a small Cas9 orthologue derived from Campylobacter jejuni. Nat. Commun. 8, 14500 (2017). 
19. Hsu, P. D. et al. DNA targeting specificity of RNA-guided Cas9 nucleases. Nat. Biotechnol. 31, 827-32 (2013).

20. Mali, P. et al. CAS9 transcriptional activators for target specificity screening and paired nickases for cooperative genome engineering. Nat. Biotechnol. 31, 833-838 (2013).

21. Fu, Y. et al. Quantification of clustered, regularly interspaced short palindromic repeats (CRISPR)-based editing system specificity. Sci. Exch. 6, 2013 (2013).

22. Pattanayak, V. et al. High-throughput profiling of off-target DNA cleavage reveals RNAprogrammed Cas9 nuclease specificity. Nat. Biotechnol. 31, 839-843 (2013).

23. Young, J. et al. CRISPR-Cas9 Editing in Maize: Systematic Evaluation of Off-target Activity and Its Relevance in Crop Improvement. Sci. Rep. 9, 6729 (2019).

24. Xie, K., Zhang, J. \& Yang, Y. Genome-wide prediction of highly specific guide RNA spacers for CRISPR-Cas9-mediated genome editing in model plants and major crops. Mol. Plant 7, 923-6 (2014).

25. Kumar, R., Kaur, A., Pandey, A., Mamrutha, H. M. \& Singh, G. P. CRISPR-based genome editing in wheat: a comprehensive review and future prospects. Mol. Biol. Rep. 46, 3557-3569 (2019).

26. Elliott, B., Richardson, C., Winderbaum, J., Nickoloff, J. A. \& Jasin, M. Gene conversion tracts from double-strand break repair in mammalian cells. Mol. Cell. Biol. 18, 93-101 (1998).

27. Gaudelli, N. M. et al. Programmable base editing of A $\bullet T$ to $\mathrm{G} \cdot \mathrm{C}$ in genomic DNA without DNA cleavage. Nature 1-27 (2017). doi:10.1038/nature24644

28. Komor, A. C., Kim, Y. B., Packer, M. S., Zuris, J. A. \& Liu, D. R. Programmable editing of a target base in genomic DNA without double-stranded DNA cleavage. Nature 533, 420-424 (2016).

29. Komor, A. C. et al. Improved base excision repair inhibition and bacteriophage Mu Gam protein yields C:G-to-T:A base editors with higher efficiency and product purity. Sci. Adv. 3, eaao4774 (2017).

30. Paquet, D. et al. Efficient introduction of specific homozygous and heterozygous mutations using CRISPR/Cas9. Nature 1-18 (2016). doi:10.1038/nature17664

31. Anzalone, A. V. et al. Search-and-replace genome editing without double-strand breaks or donor DNA. Nature 576, 149-157 (2019).

32. Shen, M. W. et al. Predictable and precise template-free CRISPR editing of pathogenic variants. Nature 563, 646-651 (2018).

33. Richardson, C. D., Ray, G. J., DeWitt, M. A., Curie, G. L. \& Corn, J. E. Enhancing homologydirected genome editing by catalytically active and inactive CRISPR-Cas9 using asymmetric donor DNA. Nat. Biotechnol. 34, 339-344 (2016).

34. Moreno-Mateos, M. A. et al. CRISPR-Cpf1 mediates efficient homology-directed repair and temperature-controlled genome editing. Nat. Commun. 8, 2024 (2017).

35. Fonfara, I. et al. Phylogeny of Cas9 determines functional exchangeability of dual-RNA and Cas9 among orthologous type II CRISPR-Cas systems. Nucleic Acids Res. 42, 2577-2590 (2014).

36. Wiktor, J., Lesterlin, C., Sherratt, D. J. \& Dekker, C. CRISPR-mediated control of the bacterial 
initiation of replication. Nucleic Acids Res. gkw214 (2016). doi:10.1093/nar/gkw214

37. Lino, C. A., Harper, J. C., Carney, J. P. \& Timlin, J. A. Delivering CRISPR: a review of the challenges and approaches. Drug Deliv. 25, 1234-1257 (2018).

38. Anders, C., Bargsten, K. \& Jinek, M. Structural plasticity of PAM recognition by engineered variants of the RNA-guided endonuclease Cas9. Mol. Cell 61, 895-902 (2016).

39. Hirano, S., Nishimasu, H., Ishitani, R. \& Nureki, O. Structural Basis for the Altered PAM Specificities of Engineered CRISPR-Cas9. Mol. Cell 61, 886-894 (2016).

40. Nishimasu, H. et al. Engineered CRISPR-Cas9 nuclease with expanded targeting space. Science 361, 1259-1262 (2018).

41. Kleinstiver, B. P. et al. Broadening the targeting range of Staphylococcus aureus CRISPR-Cas9 by modifying PAM recognition. Nat. Biotechnol. 1-7 (2015). doi:10.1038/nbt.3404

42. Kleinstiver, B. P. et al. Engineered CRISPR-Cas9 nucleases with altered PAM specificities. Nature 523, 481-485 (2015).

43. Walton, R. T., Christie, K. A., Whittaker, M. N. \& Kleinstiver, B. P. Unconstrained genome targeting with near-PAMless engineered CRISPR-Cas9 variants. Science eaba8853 (2020).

doi:10.1126/science.aba8853

44. Miller, S. M. et al. Continuous evolution of SpCas9 variants compatible with non-G PAMs. Nat. Biotechnol. 38, 471-481 (2020).

45. Karvelis, T., Young, J. K. \& Siksnys, V. A pipeline for characterization of novel Cas9 orthologs. Methods Enzymol. 616, 219-240 (2019).

46. Chylinski, K., Makarova, K. S., Charpentier, E. \& Koonin, E. V. Classification and evolution of type II CRISPR-Cas systems. Nucleic Acids Research 42, 6091-6105 (2014).

47. Chylinski, K., Le Rhun, A. \& Charpentier, E. The tracrRNA and Cas9 families of type II CRISPR-Cas immunity systems. RNA Biol. 10, 726-737 (2013).

48. Briner, A. E. et al. Guide RNA Functional Modules Direct Cas9 Activity and Orthogonality. Mol. Cell 9, 1-7 (2014).

49. Marshall, R. et al. Rapid and Scalable Characterization of CRISPR Technologies Using an E. coli Cell-Free Transcription-Translation System. Mol. Cell 69, 146-157.e3 (2018).

50. Karvelis, T. et al. Rapid characterization of CRISPR-Cas9 protospacer adjacent motif sequence elements. Genome Biol. 16, 253 (2015).

51. Harrington, L. B. et al. A thermostable Cas9 with increased lifetime in human plasma. Nat. Commun. 8, 1424 (2017).

52. Zimmermann, L. et al. A Completely Reimplemented MPI Bioinformatics Toolkit with a New HHpred Server at its Core. J. Mol. Biol. 430, 2237-2243 (2018).

53. Anderson, I. et al. Complete genome sequence of Nitratifractor salsuginis type strain (E9I37-1T). Stand. Genomic Sci. 4, 322-330 (2011). 
54. Yourik, P., Fuchs, R. T., Mabuchi, M., Curcuru, J. L. \& Robb, G. B. Staphylococcus aureus Cas9 is a multiple-turnover enzyme. RNA 25, 35-44 (2019).

55. Lee, C. M., Cradick, T. J. \& Bao, G. The Neisseria meningitidis CRISPR-Cas9 System Enables Specific Genome Editing in Mammalian Cells. Mol. Ther. 24, 645-654 (2016).

56. Sun, W. et al. Structures of Neisseria meningitidis Cas9 Complexes in Catalytically Poised and Anti-CRISPR-Inhibited States. Mol. Cell 76, 938-952.e5 (2019).

57. Paez-espino, D. et al. CRISPR Immunity Drives Rapid Phage Genome Evolution in Streptococcus thermophilus. MBio 6, 1-9 (2015).

58. Pyenson, N. C., Gayvert, K., Varble, A., Elemento, O. \& Marraffini, L. A. Broad Targeting Specificity during Bacterial Type III CRISPR-Cas Immunity Constrains Viral Escape. Cell Host Microbe 22, 343-353.e3 (2017).

59. Dong, L. et al. An anti-CRISPR protein disables type V Cas12a by acetylation. Nat. Struct. Mol. Biol. 26, 308-314 (2019).

60. Esvelt, K. M. et al. Orthogonal Cas9 proteins for RNA-guided gene regulation and editing. Nat. Methods 10, 1116-1121 (2013).

61. Morgan, S. L. et al. Manipulation of nuclear architecture through CRISPR-mediated chromosomal looping. Nat. Commun. 8, 15993 (2017).

62. Edgar, R. C. PILER-CR: fast and accurate identification of CRISPR repeats. BMC Bioinformatics 8, 18 (2007).

63. Edgar, R. C. MUSCLE: multiple sequence alignment with high accuracy and high throughput. Nucleic Acids Res. 32, 1792-1797 (2004).

64. Eddy, S. R. Profile hidden Markov models. Bioinformatics 14, 755-63 (1998).

65. Eddy, S. R. Accelerated Profile HMM Searches. PLoS Comput. Biol. 7, e1002195 (2011).

66. Chen, I.-M. A. et al. IMG/M v.5.0: an integrated data management and comparative analysis system for microbial genomes and microbiomes. Nucleic Acids Res. 47, D666-D677 (2019).

67. Nishimasu, H. et al. Crystal Structure of Cas9in Complex with Guide RNA and Target DNA. Cell 1-15 (2014). doi:10.1016/j.cell.2016.04.003

68. Kumar, S., Stecher, G. \& Tamura, K. MEGA7: Molecular Evolutionary Genetics Analysis Version 7.0 for Bigger Datasets. Mol. Biol. Evol. 33, 1870-1874 (2016).

69. Makarova, K. S. et al. Evolutionary classification of CRISPR-Cas systems: a burst of class 2 and derived variants. Nat. Rev. Microbiol. 18, 67-83 (2020).

70. Saitou, N. \& Nei, M. The neighbor-joining method: a new method for reconstructing phylogenetic trees. Mol. Biol. Evol. 4, 406-25 (1987).

71. Zuckerkandl, E. \& Pauling, L. Evolutionary divergence and convergence in proteins. Evol. genes proteins 97, 97-166 (1965).

72. Deltcheva, E. et al. CRISPR RNA maturation by trans-encoded small RNA and host factor 
RNase III. Nature 471, 602-607 (2011).

73. Karvelis, T. et al. crRNA and tracrRNA guide Cas9-mediated DNA interference in Streptococcus thermophilus. RNA Biol. 10, 841-51 (2013).

74. Markham, N. R. \& Zuker, M. UNAFold: software for nucleic acid folding and hybridization. Methods Mol Biol 453, 3-31 (2008).

75. Darty, K., Denise, A. \& Ponty, Y. VARNA: Interactive drawing and editing of the RNA secondary structure. Bioinformatics 25, 1974-1975 (2009).

76. Altschul, S. F., Gish, W., Miller, W., Myers, E. W. \& Lipman, D. J. Basic local alignment search tool. J. Mol. Biol. 215, 403-410 (1990).

77. Fu, L., Niu, B., Zhu, Z., Wu, S. \& Li, W. CD-HIT: accelerated for clustering the next-generation sequencing data. Bioinformatics 28, 3150-2 (2012).

78. Katoh, K. \& Standley, D. M. MAFFT Multiple Sequence Alignment Software Version 7: Improvements in Performance and Usability. Mol. Biol. Evol. 30, 772-80 (2013).

79. Lorenz, R. et al. ViennaRNA Package 2.0. Algorithms Mol. Biol. 6, 26 (2011).

80. Nawrocki, E. P. \& Eddy, S. R. Infernal 1.1: 100-fold faster RNA homology searches. Bioinformatics 29, 2933-2935 (2013).

81. Karvelis, T. et al. PAM recognition by miniature CRISPR-Cas12f nucleases triggers programmable double-stranded DNA target cleavage. Nucleic Acids Res. (2020).

doi:10.1093/nar/gkaa208

82. Stormo, G. D. Modeling the specificity of protein-DNA interactions. Quant. Biol. 1, 115-130 (2013).

83. Crooks, G. E. WebLogo: A Sequence Logo Generator. Genome Res. 14, 1188-1190 (2004).

84. Altschul, S. F. et al. Gapped BLAST and PSI-BLAST: a new generation of protein database search programs. Nucleic Acids Res. 25, 3389-402 (1997).

85. Mitchell, A. L. et al. MGnify: the microbiome analysis resource in 2020. Nucleic Acids Res. 48, D570-D578 (2020).

86. Frickey, T. \& Lupas, A. N. Phylogenetic analysis of AAA proteins. J. Struct. Biol. 146, 2-10 (2004).

87. Capella-Gutiérrez, S., Silla-Martínez, J. M. \& Gabaldón, T. trimAl: a tool for automated alignment trimming in large-scale phylogenetic analyses. Bioinformatics 25, 1972-3 (2009).

88. Nguyen, L.-T., Schmidt, H. A., von Haeseler, A. \& Minh, B. Q. IQ-TREE: a fast and effective stochastic algorithm for estimating maximum-likelihood phylogenies. Mol. Biol. Evol. 32, 268-74 (2015). 89. Greenough, L. et al. Adapting capillary gel electrophoresis as a sensitive, high-throughput method to accelerate characterization of nucleic acid metabolic enzymes. Nucleic Acids Res. 44, e15-e15 (2016).

90. Magnusson, A. O. et al. nanoDSF as screening tool for enzyme libraries and biotechnology 
bioRxiv preprint doi: https://doi.org/10.1101/2020.04.29.066654; this version posted April 30, 2020. The copyright holder for this preprint (which was not certified by peer review) is the author/funder, who has granted bioRxiv a license to display the preprint in perpetuity. It is made available under aCC-BY-NC-ND 4.0 International license.

development. FEBS J. 286, 184-204 (2019). 


\section{FIGURES}

A

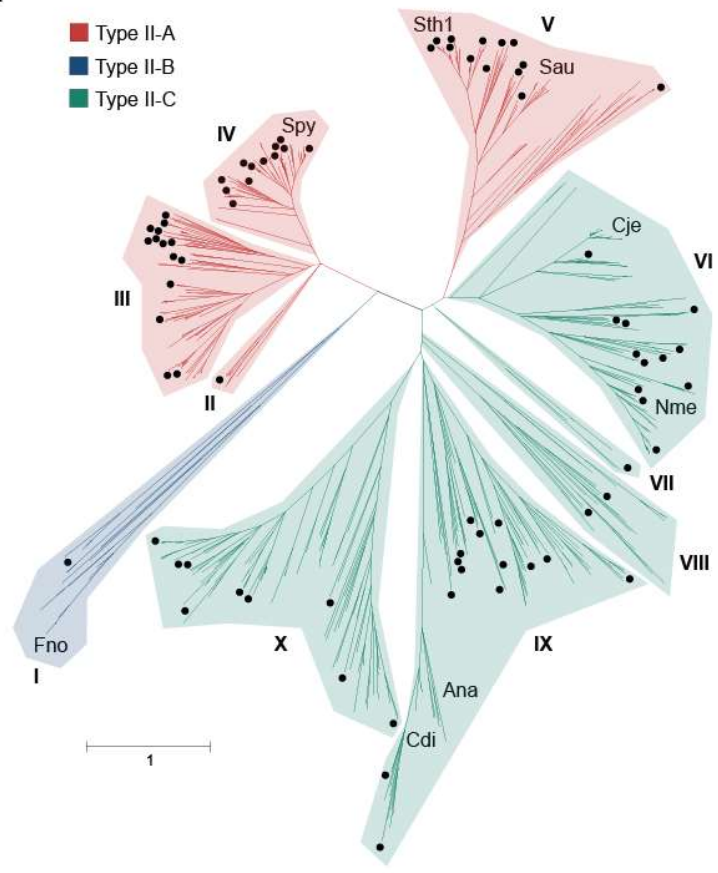

B
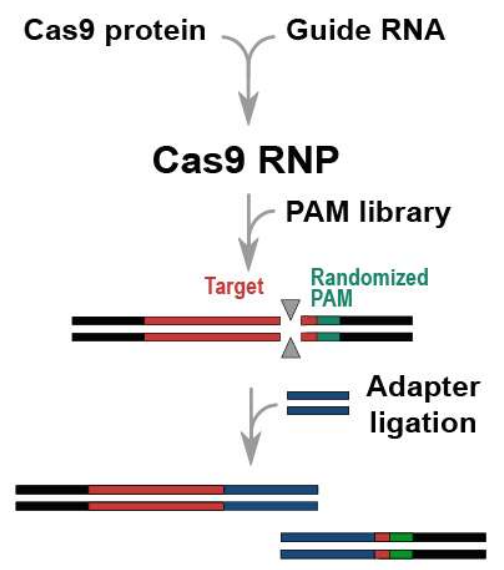

PAM fragment enrichment

\section{Deep Sequence}

\section{Figure 1.}

Cas9 diversity and characterization approach. (A) Phylogenetic representation of the diversity provided by Cas 9 orthologs. Type II-A, B, and C systems are color-coded, red, blue, and green, respectively. Distinct phylogenetic clades are numbered I-X. Those selected for study are indicated with a black dot. Cas9s whose structure has been determined are also designated. (B) Biochemical approach used to directly capture target cleavage and assess PAM recognition. Experiments were assembled using Cas9 protein produced by IVT. 


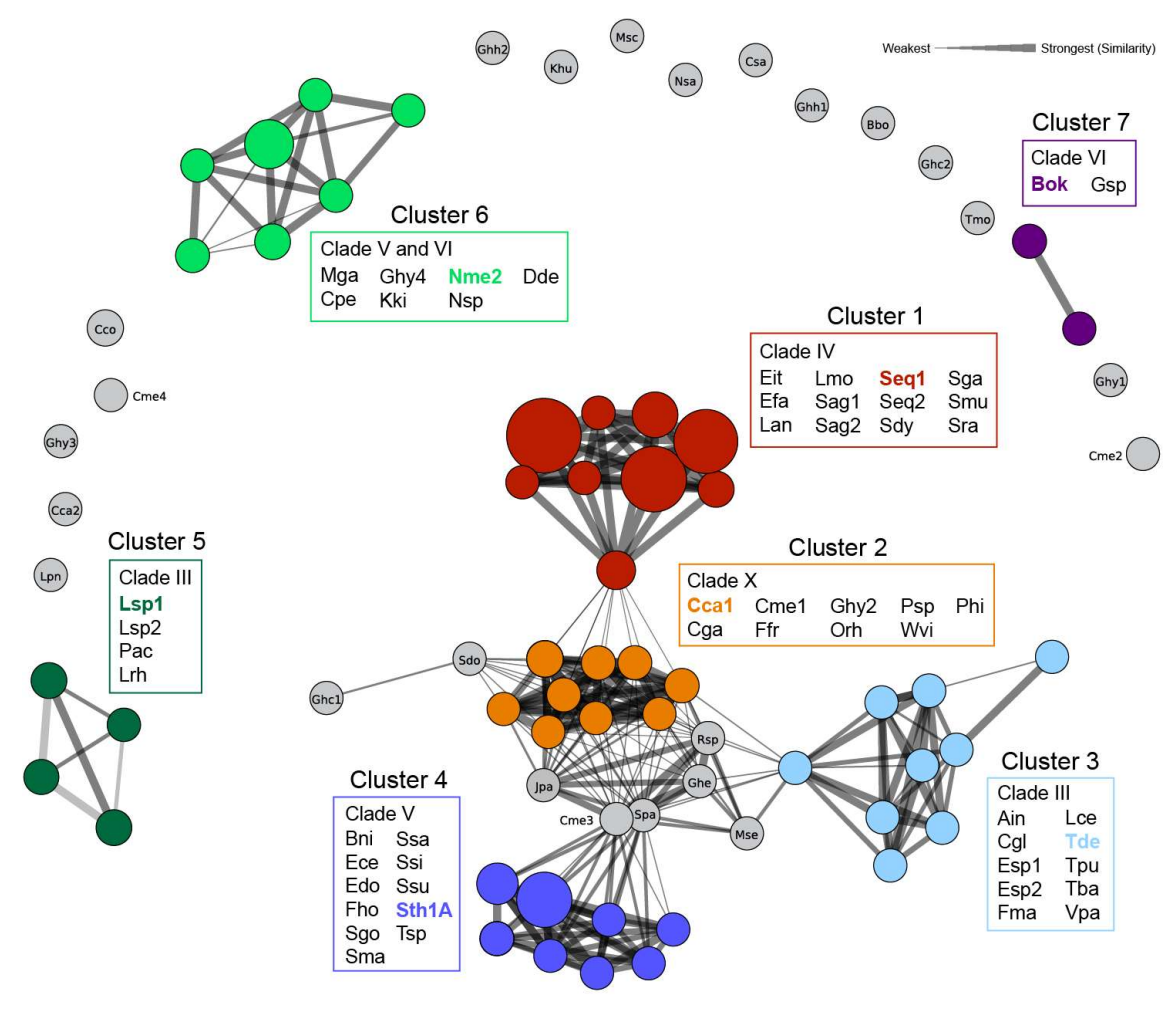

\section{Figure 2.}

Cas9 tracrRNA sequence and secondary structure similarity. Circles are scaled based on the number of sequences belonging to each covariance model (CM) and colored according to the designated cluster. The width of the connecting lines indicates the percentage of similarity or relatedness among CMs.

Representative tracrRNAs from each cluster are indicated with the associated color. CMs not assigned to a cluster are in grey. 


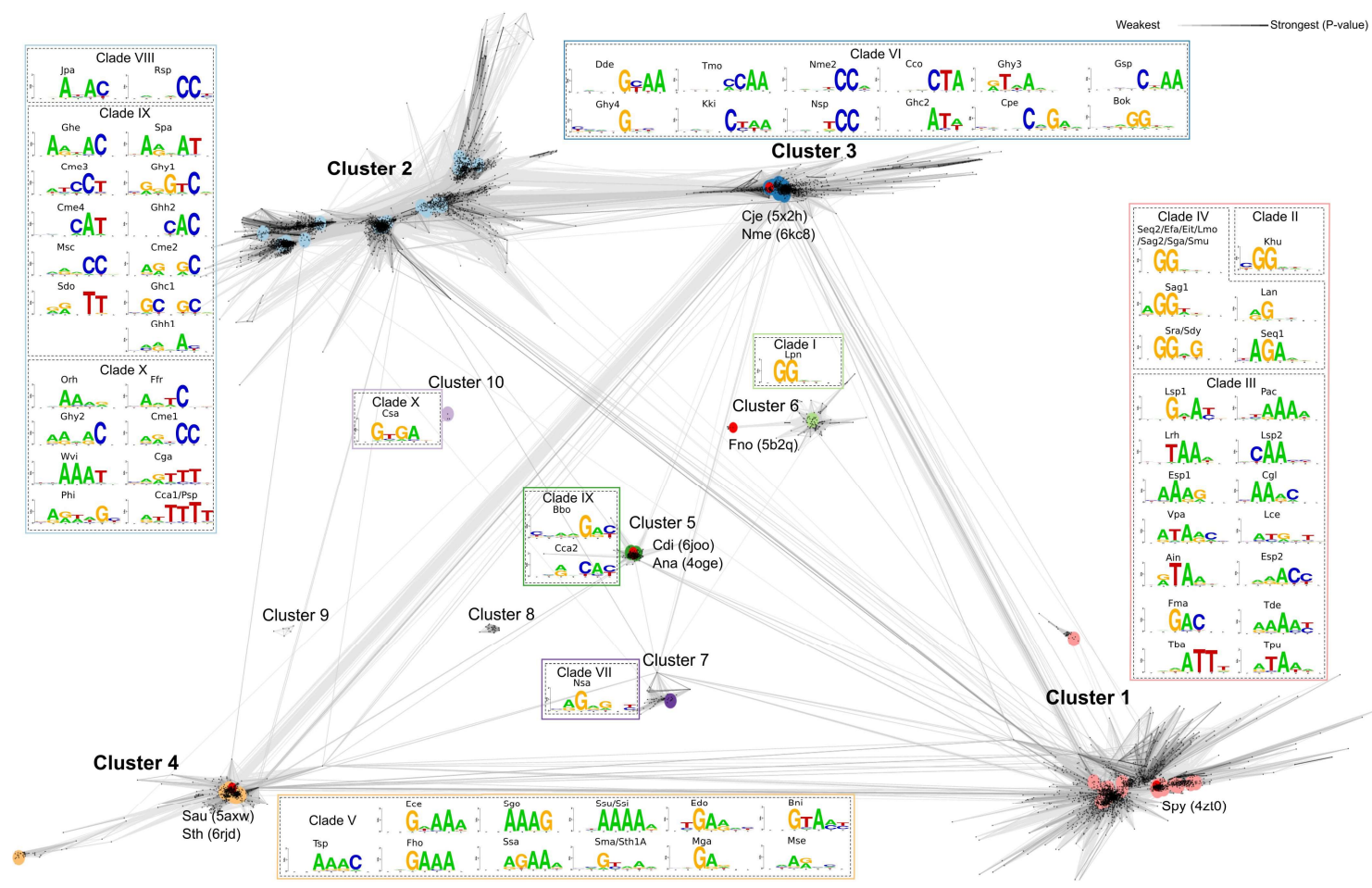

\section{Figure 3.}

Cas9 PAM Interacting (PI) domain similarity. Cas9 PI domains clustered by their pairwise sequence similarity. Lines connect sequences with $\mathrm{P}$-value $\leq 1 \mathrm{e}-11$. Line shading corresponds to P-values according to the scale in the top-right corner (light and long lines connect distantly related sequences). Major clusters are shown in bold. Cluster 1 was so named to emphasize that it contains first experimentally characterized Cas9. Clusters 2 to 10 were named beginning from the one with the most members. Sequences having known structures are marked red, their PDB code is shown in parentheses. 
A

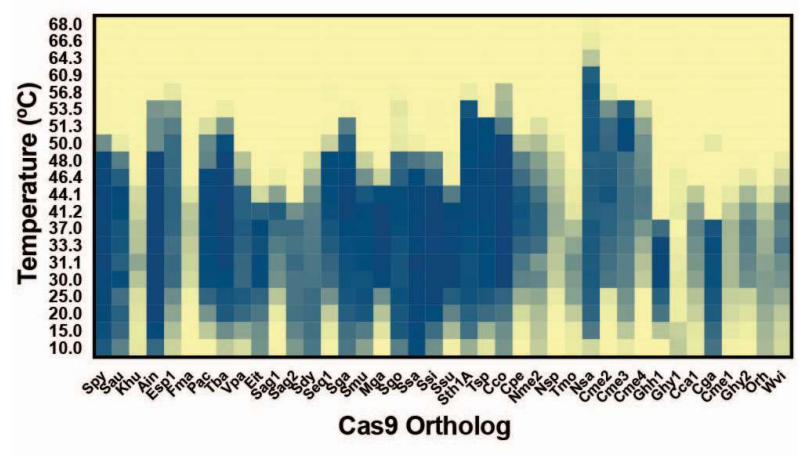

B
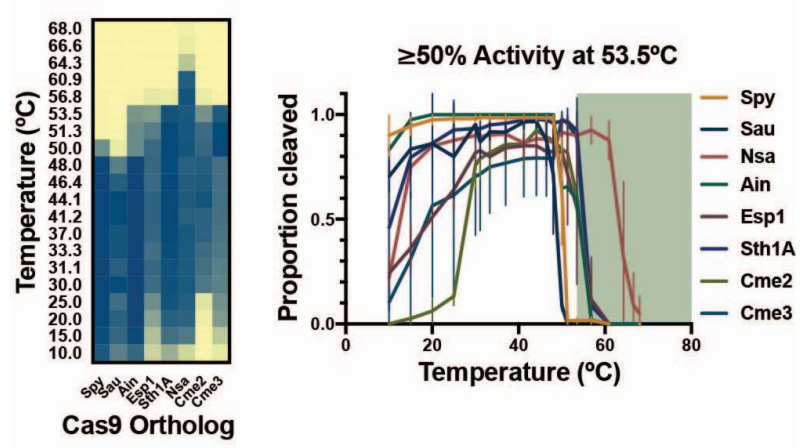

C

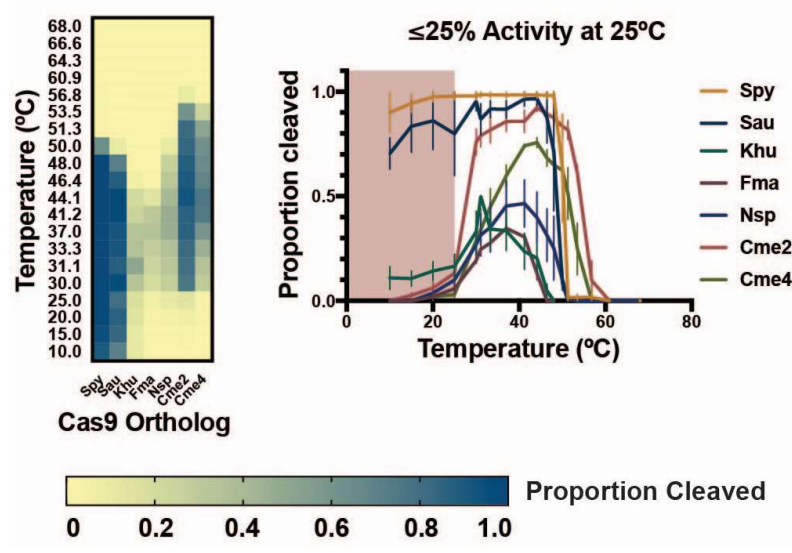

\section{Figure 4.}

Activity of Cas9 orthologs at varying temperatures. The cleavage activity of Cas9 orthologs was measured using in vitro DNA cleavage assays using fluorophore-labeled dsDNA substrates. Cleaved fragments were quantitated and are represented in a heat map (A) showing overall activity at temperatures ranging from $10^{\circ} \mathrm{C}$ to $68^{\circ} \mathrm{C}$. (B) Cas9 orthologs with activity at elevated temperatures. In vitro DNA cleavage activity for a subset of Cas 9 orthologs with $>50 \%$ activity at $53^{\circ} \mathrm{C}$ is summarized in a heat map and plotted as proportion of DNA substrate cleaved at varied temperature. Points represent the mean $+/-$ SEM of at least 3 independent experiments. (C) Cas 9 orthologs with reduced activity at room temperature. In vitro DNA cleavage activity for a subset of Cas 9 orthologs with $<25 \%$ activity at $25^{\circ} \mathrm{C}$ is 
bioRxiv preprint doi: https://doi.org/10.1101/2020.04.29.066654; this version posted April 30, 2020. The copyright holder for this preprint (which was not certified by peer review) is the author/funder, who has granted bioRxiv a license to display the preprint in perpetuity. It is made available under aCC-BY-NC-ND 4.0 International license.

summarized in a heat map and plotted as a proportion of DNA substrate cleaved at varied temperature. Points represent the mean $+/$ - SEM of at least 3 independent experiments. 
A

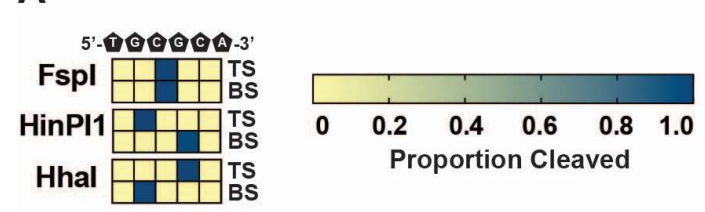

B

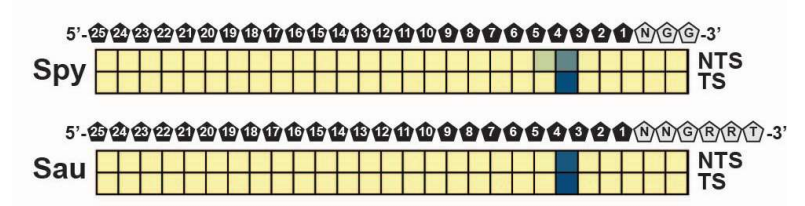

C

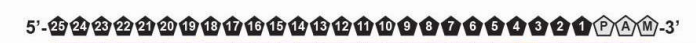

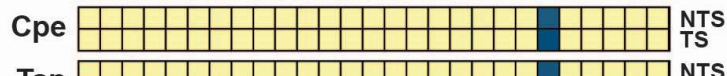
Tsp

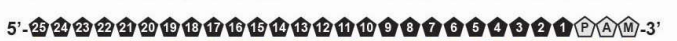
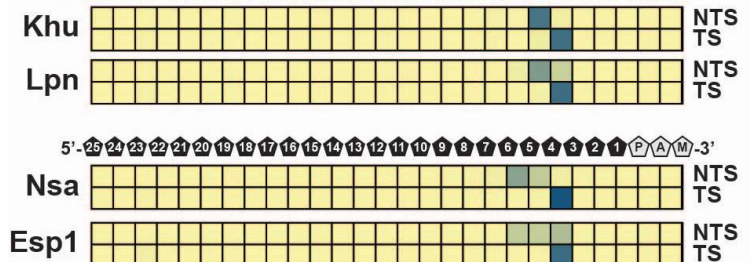

\section{Figure 5.}

Target DNA cleavage patterns produced by Cas9 orthologs. Cleavage sites and resultant dsDNA ends are depicted as heatmaps that show the proportion of cleaved ends recovered by DNA sequencing at each position of a target DNA. Intensity of the blue color indicates the proportion of mapped cleavage ends. (A) Control digests using restriction enzymes recover 5'-overhangs, 3'-overhangs, and blunt ends. TS indicates top strand; BS indicates bottom strand. (B) SpyCas9 and SauCas9 cleaved DNA ends. Heatmaps represent mapped cleavage ends as the averages at each position in 5 different dsDNA targets. Position of the DNA bases and PAM sequences is depicted above the heatmaps. NTS indicates non-target strand; TS indicates target strand. (C) Blunt and staggered-end cleavage. Examples of blunt, one base 5'-overhang staggered cleavage, and multiple base 5'-overhang cleavage are depicted as heatmaps that show the proportion of cleaved ends as the averages at each position in 5 different dsDNA targets. Position of the DNA bases and PAM sequences is depicted above the heatmaps. NTS indicates non-target strand; TS indicates target strand. 